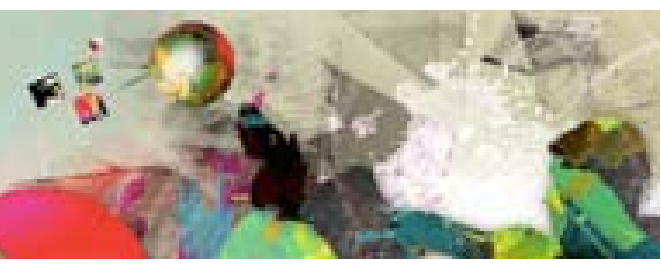

\title{
Entre certezas y dilemas: investigación sobre la experiencia de los equipos de adopción en Italia
}

\section{Between certainties and dilemmas: research on the experience of adoption teams in Italy}

\author{
Elena Allegri ${ }^{(*)}$ \\ Università del Piemonte Orientale “Amedeo Avogadro” - Italia \\ elena.allegri@sp.unipmn.it
}

\begin{abstract}
Resumen
La contribución presenta una investigación empírica sobre la experiencia de los equipos para las adopciones nacionales e internacionales en Piamonte (región del Noroeste de Italia) veinte años después de su institución. Las entrevistas y los focus groups -dirigidos a un muestreo de 48 profesionales que trabajan en la evaluación de parejas que desean adoptar- han sido orientados al estudio de sus opiniones con respecto al proceso decisorio, prestando atención a los temas tratados, a los criterios de evaluación y a las representaciones de la parentalidad adoptiva. Los resultados de la investigación confirman tanto la incidencia de las representaciones de los profesionales en el proceso decisional-que es a menudo el resultado de una me-
\end{abstract}

\begin{abstract}
The article describes empirical research on the experience of the teams dealing with adoptions in Piedmont (north-west Italy), since they were set up a little more than twenty years ago. Interviews and focus groups were conducted with a representative sample of 48 social work professionals involved in the assessment of couples wanting to adopt. The interviews focused on the themes covered in assessments, on the decision-making process, on the assessment criteria, and on the representations of what an adoptive parent is. Findings confirm the importance of social workers' representations in the decision-making process, which is often a mediation between different representations. They also show the need for a new parental identity, and system-
\end{abstract}


diación entre distintas representacionescomo la necesidad de innovar algunas perspectivas de intervención que podrían ser dirigidas más a la maduración de una nueva identidad parental y a un sistemático enfrentamiento entre todos los actores que interaccionan en el espacio social de las prácticas adoptivas.

Palabras clave: investigación, adopción, representaciones, criterio de valoración. atic dialogue between all the social actors who interact in the social space of adoption practice.

Keywords: research, adoption, representations, assessment criteria. 


\section{INTRODUCCIÓN}

A veinte años de la institución de los equipos para las adopciones en Italia ${ }^{1}$, la presente investigación tiene el propósito de reconstruir la valiosa experiencia adquirida por los profesionales que trabajan en la región del Piamonte (Noroeste de Italia), estudiando los procesos decisorios y los modelos organizativos del trabajo que los expertos activaron en toda la región en 2006 (Allegri, Favretto, Vaiuso, Bella, 2006). Entre incertezas y dilemas, psicólogos y asistentes sociales son llamados a ejercer un papel de orientadores y, al mismo tiempo, de evaluadores de las parejas que se proponen para la adopción. Por una parte, ellos pueden bien entender y compartir las motivaciones que han llevado a las personas a tomar la decisión de adoptar, por otra parte, también deben ejercer un papel estimativo cuando tengan que comprobar, antes de la decisión definitiva del juez, la efectiva capacidad de los cónyuges para "educar, instruir y mantener a los menores que pretenden adoptar". Tal valoración, no puede construirse exclusivamente sobre la existencia de algunos requisitos objetivos, exige una referencia, un cuadro teórico-conceptual, inherente a las competencias parentales y a las concretas características de la parentalidad adoptiva. En este sentido hay que destacar que también las representaciones subjetivas y sociales acerca de la parentalidad, de la parentalidad adoptiva y de la infancia, de las que cada profesional y cada juez es portador en sí mismo, concurren a formar el cuadro de referencia útil para valorar la disponibilidad y la idoneidad de las parejas que solicitan adoptar. Decidir a priori si una pareja es efectivamente capaz de ejercer con eficacia la parentalidad en el terreno adoptivo es, evidentemente, complejo: entre factores predictivos y protectores sobre el riesgo de fracaso en la adopción no existen actualmente pronósticos ciertos, objetivos y controlables en el

1 En 1985 fue adoptado el primer protocolo operativo y metodológico sobre la adopción en Italia y, en 1986 fueron instituidos por la Región del Piamonte equipos para las adopciones, con una norma con-junta de las Concejalías de Asistencia y de Salud, aprobada de acuerdo con el Tribunal para los Menores de edad. Los equipos supra-zonales, compuestos por diferentes profesionales, asistentes sociales, psicólogos y algunos neuro-psiquiatras, son actualmente 22 y colaboran con asociaciones de voluntariado y con entes competentes en la adopción internacional, de acuerdo a lo prescrito en la Convención de la Haya de 1993, ratificada en Italia con la ley $\mathrm{n}^{\circ} 476$ del 31 de diciembre de 1998.

2 Cfr. art. 6, apartado 2, Ley italiana $\mathrm{n}^{\circ} 149$ del 28 de marzo de 2001, que ha aportado algunas modificaciones a la ley del 4 de mayo de 1983, n 184, "Disciplina de la adopción y otorgamiento de la tutela de menores”, así como al título VIII del libro primero del código civil italiano, sancionando claramente el derecho del menor a una familia.

Mediaciones Sociales, № 10, I semestre 2012, pp. 89-117. ISSN electrónico: 1989-0494.

DOI: http://dx.doi.org/10.5209/rev_MESO.2012.n10.39683 
«Entre certezas y dilemas: investigación sobre la experiencia de los equipos de...»

tiempo. Además, debe de considerarse que al tratarse de un trabajo inmaterial, caracterizado por un alto grado de incertidumbre, su resultado final también depende de variables difícilmente controlables porque las específicas interacciones entre ellas se modifican y se re-determinan durante largos arcos temporales. Algunas preguntas y dilemas caracterizan el debate actual sobre el fenómeno adoptivo y han configurado el cuadro de fondo de la investigación ¿es posible crear un recorrido con las parejas dispuestas a la adopción que sea al mismo tiempo cognoscitivo/evolutivo y estimativo? ¿Cuáles son los temas que los profesionales consideran importantes en la actuación? ¿Qué elementos consideran y qué criterios se aplican para componer un cuadro estimativo lo suficiente completo y riguroso? Debido a la ingente cantidad de informaciones recogidas en la investigación, en este trabajo se presentarán los resultados más significativos de los primeros análisis exploratorios. La investigación fue encargada por la Región del Piamonte - Dirección de las Políticas Sociales y por la Agencia Regional para las Adopciones Internacionales al Departamento de Investigación Social de la Universidad del Piamonte Oriental "Amedeo Avogadro" y se realizó en el año 2005-2006. Los resultados de dicha investigación se presentaron en el simposio nacional "Apprendere dall'esperienza - Attese, realtà e prospettive dell'adozione nazionale e internazionale" ("Aprender de la experiencia - Expectativas, realidad y perspectivas de la adopción nacional e internacional") en Torino en noviembre de 2007.

Este artículo busca, en última instancia, contribuir al debate sobre las adopciones y ayudar a integrar los esfuerzos de los diferentes actores sociales que participan de él.

La importancia de este estudio reside también en la tentativa de concurrir al conocimiento del fenómeno adoptivo: es evidente que una esmerada evaluación inicial de las parejas aspirantes a la adopción puede prevenir fracasos futuros de acoplamiento. Sin embargo, no pasa inadvertido un límite ínsito en este estudio, es decir que la estructura de la investigación prevé el análisis de la perspectiva de los profesionales, pero no la de los padres adoptivos y de los hijos adoptados, que podrá incluirse en una próxima investigación. 


\section{OBJETO Y OBJETIVOS DE INVESTIGACIÓN}

El objeto del análisis se constituye no sólo por los temas investigados y los criterios de valoración aplicados por los profesionales durante el proceso de conocimiento y valoración de las parejas dispuestas a la adopción, sino también por las representaciones difusas e implícitas que influencian constantemente los pensamientos y las acciones de los profesionales. En efecto, el estudio de las fases del proceso decisional es un objetivo que exige particular atención en la reconstrucción del sentido atribuido a la acción profesional por parte de los actores implicados. Además, se debe considerar que las prácticas de integración en el proceso decisional entre profesionales también son influenciadas por la estructura, las finalidades y las rutinas de las organizaciones donde los grupos de trabajo actúan. Es notorio que dentro de los cambios sociales y de los comportamientos rutinarios (Goffman, 1969) que caracterizan la vida cotidiana, es posible localizar los mecanismos de producción y reproducción del sentido común, además de los procesos materiales y simbólicos de influencia, de disenso, de asentimiento, de conflicto, de mediación que conducen las interacciones entre actores sociales. Por lo tanto, la realidad puede ser entendida como una "construcción social” (Berger, Luckmann, 1969). Por este motivo se ha tenido en consideración la reconstrucción del pensamiento de los entrevistados respecto a las razones de su labor y a la estructuración de sus propias evaluaciones. Los interrogantes cognitivos que han orientado la investigación se refieren a dos temas: el primer tema nos interroga sobre las posibles diferencias en la representación de los profesionales sobre parentalidad biológica y parentalidad adoptiva; el segundo tema se refiere a la posible diferencia de representación de los profesionales en cuanto a adopción nacional e internacional.

\section{FUNDAMENTOS TEÓRICOS: LA PARENTALIDAD ADOPTIVA}

La parentalidad es un fenómeno complejo que, sin embargo, se puede reconducir a tres dimensiones substanciales. La primera es una dimensión biológica, conexa con la transmisión de los caracteres semejantes que vincula los hijos a los padres biológicos y también a las generaciones anteriores. Sin embargo, la semejanza conexa con el registro biológico no es sólo un dato objetivo, sino también es utilizada como señal de pertenencia del niño a la familia. La segunda es una dimensión afectiva-

Mediaciones Sociales, № 10, I semestre 2012, pp. 89-117. ISSN electrónico: 1989-0494.

DOI: http://dx.doi.org/10.5209/rev_MESO.2012.n10.39683 
«Entre certezas y dilemas: investigación sobre la experiencia de los equipos de...»

cuidadora-educativa, locución que contempla el cuidado, y el cuidar a los hijos, focalizando la atención sobre sus necesidades físicas, afectivas, cognitivas, espirituales y sociales. La tercera es una dimensión históricoparadigmática, activa ya durante el embarazo, que permite conectar el nuevo nacido con el "cuerpo familiar", gozar de la riqueza de valores, tradiciones y modalidades relacionales, que constituyen los recursos del sistema familiar (Cigoli, 1992; Greco, Cantù, 2000).

En la parentalidad biológico-natural las tres dimensiones están implicadas de lleno. Por contra, la parentalidad adoptiva se construye sobre la ausencia de la dimensión procreadora, igual que la filiación adoptiva está construida sobre la ausencia, o sobre la pérdida, del cuidado parental por parte de los padres biológicos. La elaboración de esta falta, ya sea por parte del hijo o por parte de los padres adoptivos, permite la constitución de la dimensión afectiva-cuidadora-educativa y de la históricoparadigmática. Es esta elaboración que permite al niño sentirse plenamente hijo de los padres adoptivos, a pesar de ser hijo biológico de otros (Greco, Ranieri, Rosnati, 2001).

Enfocando los procesos de regulación en el sistema de adultos educadores- niño, muchos estudios formulan construcciones teóricas relevantes para definir la influencia de la relación adulto-niño sobre el desarrollo humano. Entre esos estudios destacamos uno relativo a la capacidad de respuesta del adulto para captar las señales que expresan las diferentes intenciones, motivaciones y costumbres del niño (Ainsworth et alii 1978; Bowlby, 1989). Se trata de la teoría del apego, según la cual los primeros años de la vida del niño son determinantes para alcanzar un buen equilibrio psicofísico. En particular, la teoría afirma la importancia de una relación afectiva entre un niño y un adulto que lo cría, aunque no sea su madre o su padre biológico. Esta relación constituye una base segura por el niño de hoy y por el adulto de mañana.

En los recientes estudios, cabe señalar la teoría de co-parentalidad (Mc Hall et al., 2004; Fivaz-Depeursinge, Corboz-Warnery, 2000) que ha estimulado nuevas e interesantes investigaciones sobre los diferentes modelos que las parejas co-construyen para incluir un hijo en el núcleo familiar y que, a nuestro entender, presenta posibles e interesantes conexiones con el fenómeno adoptivo. Para observar las modalidades interactivas entre pareja durante el embarazo, se han identificado algunos

Mediaciones Sociales, № 10, I semestre 2012, pp. 89-117. ISSN electrónico: 1989-0494.

DOI: http://dx.doi.org/10.5209/rev_MESO.2012.n10.39683 
criterios: calor, intimidad de la comunicación, autonomía, poder, conflicto, estrategias de problem-solving. Estos mismos criterios han sido utilizados en la observación de las modalidades interactivas en la pareja posteriormente al nacimiento del hijo, en cuanto a cooperación, competición, calor familiar, es decir, la co-paternalidad (Malagoli Togliatti, Mazzoni, 2006).

El desafío de la parentalidad adoptiva y de las familias adoptivas está en lograr integrar los aspectos de la semejanza (la construcción a una pertenencia familiar, la búsqueda de puntos en común) con los de la diferencia (falta de un mismo patrimonio genético, diversidades culturales). Se trata de construir un "pacto adoptivo" (Scabini, Cigoli, 2000), de engendrar una "empresa conjunta” entre padres e hijos (Rosnati, Ranieri, Greco, 2003), dentro de la cual es determinante el compromiso de todos los actores implicados en la realización de la unión adoptiva.

Por estas razones, la elección de adoptar a un menor implica numerosos obstáculos, por tanto el sólo deseo de crear una familia no puede constituir el único factor a tener en consideración cuando los padres quieren adoptar.

Es más, resulta prioritario activar, con la pareja o la familia interesada en la adopción, un proceso cognoscitivo-estimativo que permita comprender sus motivaciones, recursos, límites y potencialidades. Es necesario identificar cuales son los temas a investigar, qué criterios de valoración deben aplicarse a estos recorridos y cuáles son los factores predictivos a tener en consideración.

Entre otros, Zerilli y Rosa Volpe proponen un interesante examen de los estudios sobre menores adoptados, en los que se relevan como preeminentes algunos factores que influencian el proceso adoptivo: factores genéticos (vulnerabilidad), factores pre-adoptivos (abusos y descuido, institucionalización, cambios de adultos educadores), factores asociados al proceso adoptivo y a la familia adoptiva (problemas infantiles de los mismos padres adoptivos) (en Faba Viziello, Simonelli, 2004).

Para finalizar, se puede afirmar que las contribuciones anteriormente citadas coinciden en la necesidad de activar profundas y exhaustivas investigaciones sociales y psicológicas, justo porque la parentalidad 
«Entre certezas y dilemas: investigación sobre la experiencia de los equipos de...»

adoptiva se caracteriza por un notable esfuerzo a nivel emotivo, psíquico, educativo y social.

En conclusión, se matiza como la mayoría de los estudios, que aquí se presentan en síntesis, no aclaran cuales son los criterios evaluativos que aplicar en el recorrido cognoscitivo con la pareja dispuesta a la adopción. Tal situación es atribuible a la dificultad de encontrar acuerdos en la comunidad científica, entre distintas culturas profesionales $\mathrm{y}$, a nivel social, entre actores y representantes de intereses diferentes. Esta investigación pretende ofrecer una primera contribución en esta dirección.

\section{MÉTOdo Y TÉCNICAS DE INVESTIGACIÓN}

En el cometido científico, de tipo exploratorio, se requiere una especial atención a los aspectos metodológicos desde la fase de construcción del dibujo de investigación. En efecto, para representar, de la forma más realista posible, el objeto de la investigación (tan complejo, delicado, mudable y emocionalmente "fuerte") como el proceso de valoración de las parejas adoptivas, ha sido necesario contemplar numerosos y distintos puntos de vista, variedad de métodos y técnicas, tanto por la recogida y el análisis de los datos como por la composición del grupo de investigación que hizo la lectura e interpretación. Eso explica la cantidad de puntos de vista de los profesionales implicados en el proceso de adopción (asistentes sociales y psicólogos).

En la definición de la estructura metodológica de la investigación, el punto de vista de los profesionales se ha obtenido con entrevistas individuales semi-estructuradas y también a través de focus group dirigidos a una muestra representativa, como explicaremos en el siguiente punto $(4.1)$.

La elección de la entrevista semi-estructurada (4.2), basada en una huella de preguntas, parte de la necesidad de captar valoraciones y puntos de vista elaborados individualmente por los profesionales, mientras el empleo de focus group (4.4) pretende profundizar las razones de eventuales consonancias o discrasias con lo que iba surgiendo durante las entrevistas individuales. 
La base de datos de la investigación está constituida por las respuestas de las entrevistas y los focus group. En el caso de las respuestas a las preguntas cerradas, el análisis de los datos ha sido efectuado con técnicas estadísticas y con el auxilio del programa de elaboración SPSS, Statistical Package for Social Science; en el caso de las respuestas a las preguntas abiertas, análogamente a los diálogos ocurridos en los focus groups, se utilizaron las transcripciones integrales de las grabaciones auditivas. De este modo, en momentos determinados, ha sido posible efectuar un riguroso análisis del contenido de todas las respuestas dotadas a cada pregunta específica, objeto de debate y profundización por parte del grupo de investigación.

El análisis del contenido de los textos transcritos relativos a las entrevistas y a los debates en los focus groups se ha desarrollado con el soporte del software Atlas, para enriquecer el repertorio interpretativo inicial con ulteriores reflexiones y acceder de la forma más precisa posible a la perspectiva de los sujetos estudiados.

\subsection{La muestra}

En el año 2006, en Piamonte (Noroeste de Italia) eran 147 los profesionales que trabajan en el campo de la adopción: 89 eran asistentes sociales $(60,5 \%)$ y 58 psicólogos $(39,5 \%)$. La muestra se compone de 48 profesionales. De los 48 profesionales, 44 eran mujeres $(91,7 \%)$ y 4 eran hombres (8,3\%); 28 eran asistentes sociales $(58,3 \%)$ y 20 eran psicólogos $(41,7 \%)$. Además, basándose en el criterio de la experiencia laboral en el campo de las adopciones, se crearon distintas categorías en relación con las diferentes generaciones profesionales. También estos elementos distintivos han sido útiles para captar las representaciones de la parentalidad y de la parentalidad adoptiva. Los profesionales, subdivididos entre asistentes sociales y psicólogos, fueron sub-divididos aún en dos grupos, basándose en la experiencia en el campo de las adopciones, tal y como queda representado en la siguiente tabla 1. 
«Entre certezas y dilemas: investigación sobre la experiencia de los equipos de...»

Tabla 1. Distribución por profesión y experiencia de la plantilla

\begin{tabular}{|c|l|c|}
\hline n. & \multicolumn{1}{|c|}{ Papel } & \% de la muestra \\
\hline 16 & Asistente social con escasa experiencia & $33,33 \%$ \\
\hline 12 & Asistente social desde mucho con amplia experiencia & $25,00 \%$ \\
\hline 8 & Psicólogo con escasa experiencia & $16,67 \%$ \\
\hline 12 & Psicólogo con amplia experiencia & $25,00 \%$ \\
\hline $\mathbf{4 8}$ & Total muestra & $\mathbf{1 0 0} \%$ \\
\hline
\end{tabular}

Los asistentes sociales tienen una experiencia media de trabajo en el campo de las adopciones, cercana a los 9 años y los psicólogos alrededor de 13 años, así que un asistente social con 10 años de experiencia se suele equiparar a los asistentes sociales con mayor experiencia, mientras que un psicólogo con análoga experiencia se les incluirá entre los de experiencia inferior (considerando la media de los años de experiencia de los demás psicólogos).

Para completar la descripción de las características socio-demográficas de los entrevistados, se debe añadir que los sujetos interpelados tienen una edad media de 43 años (aproximadamente 41 años los asistentes sociales y 46 los psicólogos); el entrevistado más joven tiene 27 años y el mayor 60. El 43\% de los asistentes sociales y todos los psicólogos son licenciados; tienen diploma de licenciatura el $57 \%$ de los asistentes sociales ${ }^{3}$. El "profesional tipo" es mujer. En el caso de los asistentes sociales tiene aproximadamente 41 años de edad, licenciatura y una experiencia en el campo de las adopciones de unos 9 años; en el caso de los psicólogos, tiene casi 46 años, licenciatura y una experiencia en el campo de las adopciones de unos 13 años. En ambos casos, tales datos constituyen una prueba evidente de la madurez y la experiencia profesional necesaria para afrontar temas muchos de ellos delicados y complejos.

\footnotetext{
${ }^{3}$ La dicción “diploma de licenciatura” comprende diferentes clases de diploma: diploma de Servicios Sociales y diploma de Escuelas directas con finalidades especiales, posteriormente reconvertidos en licenciatura según la normativa vigente en Italia.
} 


\subsection{La entrevista}

Los interrogantes cognitivos y los objetivos que han caracterizado la investigación se han orientado a analizar las opiniones de los profesionales ocupados en el campo adoptivo, tanto respecto a las fases y a las modalidades del proceso decisional y organizativo activadas, como en lo concerniente a los temas y los criterios estimativos y las representaciones implícitas.

Como ya se ha afirmado, la entrevista semi-estructurada dirigida a los profesionales, de una duración media de 50 minutos, ha sido conducida a partir de una huella compuesta por tres secciones. Una sección recoge datos socio-demográficos del entrevistado y datos generales relativos al equipo de pertenencia. Una sección explora los temas, los criterios y las representaciones que se refieren al proceso de valoración de las parejas. Este apartado, elaborado a través de la técnica del diferencial semántico (Osgood, 1957), incluye también el análisis de las representaciones de la parentalidad natural y adoptiva y las que se refieren al juez. Tal elección metodológica se ha revelado importante para poder reconstruir el sentido que los diferentes referentes semánticos asumen para los profesionales implicados en el proceso adoptivo.

Por fin, la tercera sección de la huella, analiza los rasgos salientes del proceso decisional individual, de la organización del trabajo, de los roles profesionales implicados, de los puntos de fuerza y de las áreas a mejorar a nivel institucional y profesional en toda la región. Las preguntas han sido de tipo cerrado, entreabierto y abierto, en razón del posible condicionamiento que un plan de cierre habría comportado en el proceso de respuesta del entrevistado.

\subsection{El diferencial semántico}

Durante la entrevista individual se les pidió a los asistentes sociales y a los psicólogos que valorasen respectivamente la figura de la madre y del padre natural, de la madre y del padre adoptivo, de la madre y del padre adoptivo internacional y del juez, eligiendo entre una serie concreta de adjetivos. Tal técnica de recogida y análisis de los datos es definida “diferencial semánticao" (Osgood, Suci, Tannenebaum, 1957) y tiene como finalidad la reconstrucción de la imagen, de la representación y del sentido que algunos objetos pueden asumir para los sujetos entrevistados.

Mediaciones Sociales, № 10, I semestre 2012, pp. 89-117. ISSN electrónico: 1989-0494.

DOI: http://dx.doi.org/10.5209/rev_MESO.2012.n10.39683 
El diferencial semántico es un instrumento "semi-proyectivo", constituido por una serie de parejas de atributos antitéticos que hacen referencia a distintas áreas de sentido, como por ejemplo, bueno/malo, activo/pasivo, triste/alegre, pesado/ligero. Cada pareja de adjetivos referidos a la unidad de análisis se valoran en una escala de 1 a 7 , donde 7 corresponde a un máximo de "bueno" y 1 a un máximo de "malo". En el caso de la investigación que aquí presentamos, la unidad de análisis se constituye por la representación de los distintos tipos de parentalidad investigados y la serie de parejas de atributos antitéticos está formada por 15 parejas de adjetivos.

Una de las características fundamentales de esta técnica consiste en intentar reconstruir el perfil de un objeto sin preguntar directamente a los entrevistados que de otro modo se arriesgarían a respuestas muy influenciadas por su propia misma cultura, por su entorno, por su misma capacidad expresiva, por el tipo de interacción establecida con el entrevistador (colaborativa, amistosa, conflictiva...) y por el momento concreto en que se les solicita la respuesta. En cambio, a través del diferencial semántico, los sujetos son invitados a responder en una escala graduada igual para todos (estímulo estandarizado) para que no olviden o ignoren ningún aspecto considerado relevante del concepto propuesto, a menos que no expresen una precisa voluntad en tal sentido. Además, para proveer una configuración o imagen del objeto propuesto, en lugar de usar palabras o frases, son utilizados números, lo cual tiene la ventaja de simplificar la comparación entre las distintas percepciones y representaciones de los entrevistados y llegar a trazar un perfil de los distintos objetos, según la representación media que procede de las respuestas. La comparación se puede desarrollar en dos direcciones, es decir, tanto respecto a los objetos a valorar, como respecto a quien evalúa, por ejemplo observando que grupos de entrevistados se han alejado más de la visión media de un concepto particular.

\subsection{Los focus group}

Objetivo implícito en los focus group ha sido la profundización de algunas de las temáticas surgidas durante las entrevistas y el control de unas hipótesis de investigación notadas en las fases anteriores del trabajo. La técnica de búsqueda del focus group no prevé, entre sus asunciones de 
rigor científico, la representatividad de la muestra y la inferencia de los resultados de la muestra a la población, por tanto en la selección de los casos no se ha tratado reproducir las mismas proporciones de los variados subgrupos de profesionales. Los grupos se formaron, considerando la disponibilidad de los entrevistados, para garantizar el más alto nivel posible de homogeneidad de los participantes según la profesión (asistentes sociales, psicólogos) y según la experiencia (profesionales que trabajan desde mayor o menor tiempo). A tal fin se organizaron 5 focus groups: uno con los responsables de los varios equipos, dos con los asistentes sociales con menor y mayor experiencia, dos con los psicólogos con menor y mayor experiencia. Así pues, en los focus group han participado profesionales que representan la casi totalidad de los equipos territoriales de la región del Piamonte. En total, en los focus groups se entrevistaron 37 personas, entre la cuales 24 eran asistentes sociales, 12 en calidad de referentes de los equipos territoriales, 7 como asistentes sociales con menos experiencia y 5 con más, 13 psicólogos, 2 en calidad de referentes, 5 con menos experiencia y 6 con más experiencia.

A continuación se exponen los resultados de la búsqueda originados por las entrevistas individuales y por los focus groups.

\section{RESULTADOS Y PRIMEROS COMENTARIOS}

Para empezar la entrevista se propuso una breve historia presentada por una pareja dispuesta a la adopción ${ }^{4}$. El objetivo fue estimular cada psicólogo y asistente social, de los 48 entrevistados, a singularizar las

4 Reconducimos la breve historia de presentación de la pareja: "Paolo tiene 45 años y es dirigente en una gran empresa. Federica tiene 37 años y es profesora en un instituto de estudios superiores de la ciudad. Ambos tienen buen aspecto, están muy ocupados con el trabajo, y no obstante, logran conducir una vida llena de intereses personales y amistades. En cambio, no logran tener a un hijo. Después de algunas tentativas de inseminación artificial, han decidido presentar su candidatura para una adopción nacional e internacional. Tienen una casa confortable y adecuada en la organización de los espacios para acoger a un menor adoptado. De los coloquios con psicólogo y asistente social, y luego en la visita al domicilio, resulta claro que todavía no han madurado una idea clara del sentido de la adopción. En particular declaran no tener preferencias ni resistencias con respecto de patologías físicas y psicológicas del menor a adoptar. Además declaran no tener preferencias respecto a la etnia, o al estado de patología mental y/o de drogadicción de la madre. Los profesionales empeñados en el proceso de valoración de la pareja candidata a la adopción, no consiguen ayudar a Paolo y Federica a definirse de forma más clara”. 
informaciones fundamentales que faltaban para asumir su propia posición con respecto de la idoneidad o no, de los potenciales adoptantes. Solo 3 entrevistados se declararon inmediatamente a favor de la idoneidad de la pareja, mientras que la mayoría de los entrevistados (27 sobre 48) consideró la pareja como no idónea, o bien declaró no tener elementos suficientes como para poder decidir (18 sobre 48). Las respuestas -tanto con respecto a las informaciones que faltaban, como de los motivos útiles para argumentar la valoración de no idoneidad- se agrupan alrededor de tres temas: la parentalidad adoptiva (19 citas), la motivación a la adopción (16 citas), la relación de pareja (16 citas). A continuación, como ejemplo, unos fragmentos de las entrevistas:

"Lo que me produce perplejidad es que se acepte, de un modo tan carente de crítica, el hecho de que también pueden haber patologías fisicas y psíquicas. Normalmente, cuando se quiere a un hijo se lo imagina sano. Por tanto una pareja que acepta de modo indiscriminado este tipo de recorrido adoptivo o es una pareja que forma parte de grupos de familias con un fuerte empeño social, o bien me pregunto cuál es el deseo que los mueve o, en fin, si son conscientes de lo a que van encuentro" (entrevista $\mathrm{n}^{\circ} 6$ ).

"Falta un sentido más realista de lo que tienen que enfrentar y por lo tanto de su tarea de padres que se arriesgan en una empresa bastante complicada. ¿Será posible que no tengan temores, dificultades, miedos, y también preferencias, por qué no? Haría falta hablarlo mucho con ellos" (entrevista $\mathrm{n}^{\circ} 35$ ).

En estas primeras respuestas surge la representación de un recorrido de conocimiento y valoración de la pareja que se desenvuelve en la relación con los expertos. En este sentido, muchos profesionales han destacado la necesidad de ayudar a la pareja a conocerse a sí misma, señalándoles los puntos de fuerza y los elementos de criticidad útiles para una valoración de las competencias de la parentalidad.

\subsection{Las representaciones de la parentalidad: el diferencial semántico}

Como ya se ha presentado en el punto 4.3 , durante la entrevista individual se les preguntó a los asistentes sociales y a los psicólogos que valorasen respectivamente la figura de la madre y del padre biológico, de la madre y del padre adoptivo, de la madre y del padre adoptivo internacional y del juez, considerado el sujeto primer responsable en el proceso decisional relativo a la declaración de idoneidad de la pareja aspirante a la adopción. Los 48 profesionales entrevistados pudieron elegir

Mediaciones Sociales, № 10, I semestre 2012, pp. 89-117. ISSN electrónico: 1989-0494.

DOI: http://dx.doi.org/10.5209/rev_MESO.2012.n10.39683 
entre una lista de 15 parejas de adjetivos: "dulce-áspero”, "lejano-cercano”, "bueno-malo”, “cálido-frío”, "feo-bonito”, “débil-fuerte”, "resistente-frágil”, "rígido-flexible”, "tolerante-intolerante”, "estable-inestable”, “irracionalracional”, “rápido-lento”, "vago-exacto”, “dinámico-estático”, “previsible imprevisible”.

La intención fue construir algo como un perfil de la personalidad de los diferentes sujetos implicados para poder valorar si existieran diferencias sustanciales en las proyecciones de los profesionales. El gráfico de las respuestas medianas (gráfico 1) ilustra con claridad los resultados conseguidos. Es el fruto de la comparación entre los resultados conseguidos respecto a las representaciones de todos los profesionales sobre los varios tipos de padres indagados.

Gráfico 1. Diferenciales semánticos juicios totales

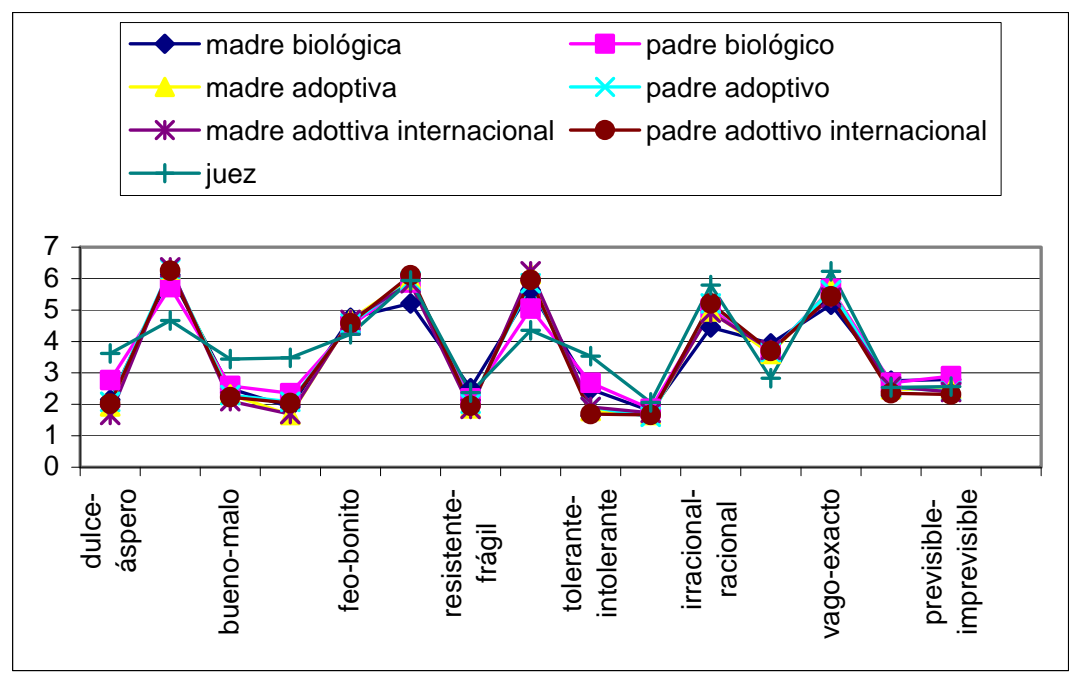

Sorprende como, en la mayoría de los casos, el conjunto de sentido de los psicólogos y los asistentes sociales se sobrepone tanto por lo que concierne los contenidos de la representación de la parentalidad natural y adoptiva, como por las representaciones de la figura del juez. 
Las áreas semánticas, localizables por la agregación de categorías de las múltiples representaciones producidas en relación a los estímulos de la entrevista, trazan el mismo horizonte de sentidos. Las respuestas a los estímulos son atribuibles a una idea general de disponibilidad afectiva, de responsabilidad consciente en educar al respeto de las reglas y confirman la correspondencia entre la representación de la parentalidad biológica y de la adoptiva, sin particulares diferencias entre adopción nacional e internacional. En efecto, como se puede notar leyendo el gráfico, las puntuaciones medianas referidas a cada ítem del diferencial semántico, resultan por la mayoría desequilibradas hacia el término positivo de la pareja de adjetivos propuestos.

En conclusión, las representaciones de parentalidad biológica y adoptiva de los asistentes sociales y de los psicólogos coinciden. Esas representaciones posiblemente se pueden atribuir a modelos de paternidad y maternidad tradicionales, considerados cruciales para asegurar un crecimiento armónico de los hijos. En efecto, los entrevistados consideran que por los hijos la conducta de los padres debe resultar previsible, es decir referible a reglas educativas claras y estables, tan como la relación afectiva.

Y por fin, por lo que atañe la representación de los profesionales sobre el papel del juez, los resultados evidencian, con distintos pesos, una relativa distancia y un cierto estatismo. Los jueces, aparecen estables, muy racionales, muy esmerados, fuertes. El resultado es atribuible a las características usualmente relacionadas con el papel de los que tienen que garantizar la certeza del derecho e indican el deseo de establecer relaciones de enfrentamientos más estables y sistematizados en el tiempo. Estas últimas consideraciones han sido confirmadas por los datos relativos a las entrevistas, como se describe en los puntos detallados a continuación.

\subsection{Los temas tratados en el proceso estimativo}

Todas las citas propuestas por los entrevistados, relativas a los argumentos que hay que afrontar en el recorrido cognoscitivo-estimativo concernientes a la adopción nacional, han sido posteriormente unidas, siguiendo el dictado legislativo italiano: "las investigaciones (...) conciernen, en particular, a la capacidad de educar al menor, la situación personal y económica, la salud, el entorno familiar de los solicitantes, las razones por las

Mediaciones Sociales, № 10, I semestre 2012, pp. 89-117. ISSN electrónico: 1989-0494.

DOI: http://dx.doi.org/10.5209/rev_MESO.2012.n10.39683 
«Entre certezas y dilemas: investigación sobre la experiencia de los equipos de...»

que los solicitantes desean tutelar al menor" (art. 22, apartado 4, 1. n. 149/2001). Por lo que concierne a la adopción internacional, y para una observación lo más completa posible, los elementos que los profesionales tienen que profundizar están previstos en la Convención de la Haya (1993) y se refieren a: la historia personal de cada cónyuge y de cada pareja, la historia familiar, la situación sanitaria, el entorno social donde se desarrolla su vida, las motivaciones que los llevan a adoptar, las capacidades y las competencias para cuidar un hijo de diferente nacionalidad y etnia, las características de los menores que serían capaz de acoger, como ya queda ilustrado en el punto 3.2 de esta elaboración.

$\mathrm{El}$ análisis de los textos de las entrevistas y los focus group, relativos a las respuestas sobre los temas tratados durante el recorrido con la pareja, evidencian en total 489 citas de temas considerados útiles en el proceso decisional para la adopción nacional e internacional. Entre estos, 372 se refieren a la adopción nacional y 117 a la internacional. Las indicaciones sobre los argumentos de estudio de la pareja han sido posteriormente unidas siguiendo el criterio de la profesión, como queda evidenciado en la siguiente tabla 2. De estas, 299 (61\%), han sido expresadas por los asistentes sociales y 190 (39\%) han sido sugeridas por los psicólogos. Esos porcentajes reflejan las proporciones de la muestra representativa de los profesionales piamonteses.

Tabla 2. Distribución por profesión de las citas de temas a explorar para la adopción nacional e internacional. Total 489

\begin{tabular}{|c|c|c|c|c|c|}
\hline Adopción nacional & a.s. & psi & $\begin{array}{l}\text { Adopción internacional } \\
\text { (contenido específico) }\end{array}$ & a.s. & psi \\
\hline El menor de edad & 66 & 42 & La integración social del menor (etnia-diferencias) & 55 & 19 \\
\hline $\begin{array}{l}\text { La parentalidad } \\
\text { adoptiva }\end{array}$ & 75 & 49 & La integración del menor en la familia ampliada & 3 & 1 \\
\hline \multirow[t]{4}{*}{ La relación de pareja } & 74 & 66 & La disponibilidad de la pareja hacia otras culturas & 16 & 10 \\
\hline & & & $\begin{array}{l}\text { La disponibilidad a viajes a lugares lejanos (capacidad de } \\
\text { adaptación) }\end{array}$ & 9 & 1 \\
\hline & & & La imagen del niño adoptivo & o & 2 \\
\hline & & & La representación de la familia de origen del niño adoptable & 1 & 0 \\
\hline \multirow[t]{2}{*}{ Total } & 215 & 157 & & 84 & 33 \\
\hline & \multicolumn{2}{|c|}{372} & & \multicolumn{2}{|c|}{117} \\
\hline TOTAL CITAS & & & 489 & & \\
\hline
\end{tabular}

Mediaciones Sociales, № 10, I semestre 2012, pp. 89-117. ISSN electrónico: 1989-0494. DOI: http://dx.doi.org/10.5209/rev_MESO.2012.n10.39683 
Los resultados evidencian una gran atención a los temas sobre los que apoya la disponibilidad a la parentalidad adoptiva. Dichos temas han sido: el menor de edad (las características subjetivas, la historia y el contexto de la vida del pasado del menor); la parentalidad adoptiva (las características subjetivas de las personas como adultos y como futuros padres y madres), los conocimientos y las competencias parentales, la capacidad para afrontar los acontecimientos traumáticos y estresantes de la vida, la elaboración del luto debido a la esterilidad (cuando exista), la relación de pareja (el tipo de estructuración de los papeles y las relaciones entre la pareja, la capacidad de enfrentar el estrés, de activar estrategias de problem solving). Por lo que concierne los temas a explorar en la adopción internacional, cada entrevistado ha mencionado solo cuestiones específicas, considerando que las que ya habían citado en tema de adopción nacional, resultaban comunes a ambos ámbitos. Los temas considerados específicos han sido: la integración social del menor (los problemas consiguientes a la etnia y a los rasgos somáticos diferentes, la integración del menor en la familia ampliada), la disponibilidad (la curiosidad y el interés por otras culturas, la disponibilidad a viajar, entendido no tanto en el sentido económico, sino como capacidad de adaptación) y por fin, otros temas que conciernen a la imagen del niño adoptable y a la representación de su familia de origen.

Por lo tanto, el empeño prodigado por los profesionales tiende a la búsqueda de todos los elementos útiles a evidenciar comportamientos y actitudes congruentes por parte de cada uno de los adoptantes en el pasaje de pareja a padres adoptivos, incluyendo acontecimientos y pasos dolorosos a recordar o considerados muy íntimos, como queda evidenciado en las entrevistas y en los focus group transcriptos a continuación, como ejemplo.

- La pareja y la esterilidad (140 citas por adopción nacional): "Tratar el tema de la esterilidad, significa entender si la pareja logra separarse de la imagen del hijo biológico, si logra separarse del dolor por este deseo imposible, si consigue pensar en un niño que adoptar y pensándolo, acompañada por los operadores, puede entrar en la óptica de un niño adoptado, así que se pueda valorar si hay una motivación por parte de ambos en la pareja" (entrevista 20). 
- La parentalidad adoptiva (124 citas por adopción nacional): "Por lo tanto, cuál ha sido el cambio de la familia, la importancia, el sentido que el hijo tiene en la pareja, mientras tanto nosotros lo hemos visto transformarse, porque una vez los hijos llegaban si llegaban. Buscar a un hijo, que no se pudo tener biológicamente, dirigirse a la adopción, respondía a posiciones ideológico-políticoreligiosas también solidarias, que tenían valores ideales muy claros, y también un buen soporte para llegar a esta clase de elecciones. Hoy en día, me parece que la búsqueda de un hijo sigue más valores personales, de derecho, narcisísticos, decimos por lo tanto de inversión hacia algo que yo quiero y tengo que conseguir a toda costa. Nos lleva a una dimensión menos abierta a lo problemático, porque quizás se coloca en el área de la necesidad más que en la del deseo, y si yo lo deseo no necesariamente voy a conseguirlo" (f.g. 23.03.2006).

- La integración social del menor con rasgos somáticos diferentes (74 citas por adopción internacional): "Qué clase de orientación tienen hacia la diversidad, también en cuanto a la etnia; no sólo por ellos, también por la familia ampliada porque en muchos casos tiene su importancia, y te das cuenta que hay resistencias también cuando los padres ya han dado consenso a la adopción internacional, pero te enteras que hay algo desentonado en lo que te han contado. Intentas ver más a estos aspectos" (entr. 14).

Los resultados indicados hasta este punto, perfilan una orientación del trabajo de evaluación de los operadores. En efecto, surge una fuerte tendencia a afrontar todos los temas contemplados por las disposiciones legislativas. En particular, resulta evidente el empeño de asistentes sociales y psicólogos en analizar las motivaciones, la disponibilidad a acoger la diversidad, las modalidades de funcionamiento de la potencial pareja de padres adoptivos, para sondear si la pareja es capaz de coger el desafío lanzado por la parentalidad adoptiva, es decir lograr integrar aspectos de identidad y semejanza virtual (la construcción de una pertenencia familiar) con los de diferencia (falta de un mismo patrimonio genético).

En el siguiente apartado podremos apreciar como se han indagado los temas afrontados, si se han utilizados criterios de valoración atendibles y cuáles son. 


\subsection{Los criterios de valoración utilizados en el proceso decisional para la adopción nacional e internacional}

En el análisis de los textos de las entrevistas se han recogido 296 citas de criterios útiles tanto por la adopción nacional, como por la internacional, sin vínculo con respecto del número de citas posibles. Las citas han sido incorporadas en grupos temáticos que hacen referencia a: relación de pareja (32 por la adopción nacional y 14 por la internacional), sensibilidad emotiva (44 por la adopción nacional y 6 por la internacional), parentalidad (38 por la adopción nacional y 27 por la internacional), competencias transversales (39 por la adopción nacional y 27 por la internacional), modelos teóricos (8 por la adopción nacional y 3 por la internacional), instrumentos (46 por la adopción nacional y 12 por la internacional), como se representa en la tabla 3.

Tabla 3. Criterios de valoración para la adopción nacional e internacional, agrupados por tema y por profesión

\begin{tabular}{|c|l|c|}
\hline $\begin{array}{c}\text { Adopción nacional } \\
\text { Frecuencia citas }\end{array}$ & Criterio que se refieren a: & $\begin{array}{c}\text { Adopción internacional } \\
\text { Frecuencia citas }\end{array}$ \\
\hline 32 & Relación de pareja & 14 \\
\hline 44 & Sensibilidad emotiva & 6 \\
\hline 38 & Parentalidad & 27 \\
\hline 8 & Modelo teórico & 12 \\
\hline 46 & Instrumentos & 27 \\
\hline 39 & Competencias transversales & \\
\hline & & 89 \\
\hline 207 & Total & \\
\hline & 296 & \\
\hline
\end{tabular}

Sorprende que el número más alto de citas (46) se refiere al tema de instrumentos y no a los criterios de valoración. Muchos entrevistados, frente a la pregunta sobre los criterios utilizados han preferido ilustrar los instrumentos profesionales (la charla, el genograma, las pruebas psicológicas, las preguntas puestas a la pareja) evitando hacer referencias concretas a criterios, también cuando el entrevistador les ha propuesto 
algunos sinonímicos (parámetros, indicadores, descriptores) como se deduce de los testimonios transcritos a continuación:

"Usted está preguntándome cosas [los criterios de evaluación] que no acabo localizar como elementos específicos; en mi opinión es un discurso global que se modula entre contenidos y actitudes, disponibilidad" (ent.28).

"El instrumento es el coloquio, no hay otros instrumentos. Hay parejas que expresan muchas dificultades a exteriorizar las emociones. El esfuerzo es el de verbalizar esta dificultad que yo percibo e invitar a la persona a explorar este aspecto. Desde luego es algo que exige tiempo y que, a lo mejor, la primera vez no lo consigues" (ent.14).

"A través de preguntas abiertas o cerradas, depende. En la mayor parte de los casos cuando he recomendado a la pareja que reflejara sobre la adopción internacional, porque evidenciábamos perplejidades y dificultades, la pareja no se ha mostrado dispuesta" (ent.7).

"Representamos un objeto definido, un niño con una historia inventada, y vemos cómo ellos mismos se ven como padres, como cuentan de ser respecto a un niño, como cada uno ve a el otro como padre o madre y luego, dotes fundamentales son la capacidad de contención, la afectividad, una buena capacidad de sujetar la frustración" (ent.6).

Muchos operadores han mencionado, como criterios de valoración, el clima emotivo de los coloquios y el grado de participación emotiva al recorrido demostrado por parte de los candidatos padres adoptivos (50 citas):

"Adviertes la afectividad, en cambio algunas veces, encuentras rigidez, mutismo; hay parejas que ni se miran durante los coloquios, otras que se cogen la mano. Quiero decir, que en el recorrido captas todo un conjunto de cosas sin dar nada por descontado. Yo estoy abierta a la comunicación; a menudo lo que pasa es que empiezo yo con las parejas y luego la psicóloga. A veces hay elementos que me molestan, así que trato racionalizar y decir "atención estoy proyectando, estoy proyectando mucho" (f.g.7.06.2006).

Muchos profesionales han señalado como cualidades indispensables en cada adulto que se acerca al fenómeno adoptivo unas capacidades y competencias: elasticidad, flexibilidad, destreza de perspectiva, capacidad de cambiar de opinión, propensión a la planificación, competencias de negociación. Por lo tanto, han sido catalogadas bajo el título de competencias transversales (66 citas): 
"Nosotros intentamos ayudarlos a madurar expectativas reales, diciéndoles que en el ámbito de la adopción internacional los niños que llegan son niños más grandecitos, son niños con determinadas problemáticas. Procuramos informarlos, de la mejor manera, también sobre el principio de subsidiariedad, pero yo considero que es más complicado valorar, hacer nuestra valoración en este ámbito. Advierto que no nos aportan muchos elementos, no tenemos muchos elementos para evaluar” (ent. 48).

Las citas relativas a los criterios aplicables en la valoración de las competencias parentales (65 citas) y a la relación de pareja (56 citas) se concentran en la capacidad de la pareja de resistir en los acontecimientos estresantes de la vida, en su capacidad de reconocer al otro como padre/madre, en su capacidad reparadora con respecto a los traumas padecidos por el menor adoptado, en las modalidades con que se enfrentan al dolor, como testimonian los pasajes de algunas entrevistas a continuación:

"Por lo general, el criterio es el paso por el dolor y como han salido de ese dolor, porque entre el niño biológico y el niño adoptivo hay un gran 'gap'. Cómo se ha vivido el pasaje por el dolor, si los ha distanciado, si los ha acercado, si es un recurso de pareja o si se ha culpabilizado el otro. Indudablemente la modalidad con la que cuentan, es decir, si son capaces de comunicar con sus palabras, me permite también entender cuántas posibilidades tendrá el niño de ser acogido" (entr.1).

"El criterio de visibilidad social no es el que yo considero; hay criterios de estabilidad, de autenticidad de las relaciones, de valoración del contexto, de inserción social. Me preocupa la pareja que dice que está muy bien, que son alegres. (...) Prefiero utilizar un criterio de integración social, que sea una asociación o la iglesia no importa, mejor que el logro de un estatus económico y social que algunas parejas te ponen delante para cerrarte la boca” (entr. 2).

Con respecto de los modelos teóricos, las citas (8) hacen referencia a enfoques teóricos distintos (modelo del apego, teorías que hacen referencia al desarrollo mental, modelo sistémico y ciclo de vida familiar) sin embargo sin precisar cuáles criterios de valoración puedan inspirar:

"Yo tengo un criterio de lectura que viene de mi formación sistémica, con lo cual mi lectura está centrada en un marco de relaciones, en el contexto donde se mueven, sobre el dónde, sobre el cuándo y sobre el quién hace qué, por lo tanto son los criterios, que yo tengo más presentes: la capacidad de entrar en empatía con el otro, de relación al comunicarse con el otro y, sobre todo, que haya un espacio, que se perciba la necesidad de espacio y de tiempo. Con 'tiempo' quiero decir ritmo, o sea que haya un tiempo justo. Uno puede llevar 10 años en madurar una cosa y 2 en superarla, 
otro la hace en 1 año y otro en 5 y otro nunca, pero aquí son dos y lo que me interesa es el ritmo, porque a veces lo que ocurre es que uno ya está bien delante y el otro detrás y el resultado es como una danza" (ent. 11).

Pues, la gran parte de los profesionales ha demostrado una fuerte tendencia a confundir criterios y temas en su misma narración. Tal incierta tendencia no puede ser atribuida sólo a los profesionales, más bien debería de considerarse en un contexto más amplio, puesto que implica cuestiones afrontadas en el debate internacional, como ha sido expuesto en el punto 3 de este elaborado.

Es notorio que valorar sin un aproche científico, comporta el riesgo de personalizar y centrar sobre si mismo cada criterio de análisis y de juicio. La consecuencia es que la valoración se convierte en una negociación entre profesionales y puede ser influenciada por la relación entre ellos, por las dinámicas de poder internas al equipo. A tal propósito, también el debate internacional, acerca de los sistemas de valoración en ámbito psico-social, todavía no ha solucionado la cuestión. Por esta razón, se ha preferido presentar los resultados del análisis de los textos transcritos, en efecto, la riqueza de los testimonios recogidos puede constituir una base provechosa para el intercambio entre los actores implicados en el fenómeno adoptivo.

Para que se note la relación de los criterios estimativos con las transformaciones sociales de las familias, se ha indagado el posible cambio ocurrido en las formas de estudiar a los aspirantes a la adopción. Los psicólogos y los asistentes sociales piamonteses afirman que ha ocurrido un cambio, no tanto con respecto de los criterios de valoración, sino más bien en las modalidades de aplicación. Con tal propósito, los profesionales entrevistados han indicado 90 citas que se refieren a los cambios ocurridos durante un tiempo y que, por sus propias peculiaridades, han sido asociadas en dos grupos. El primer grupo se refiere a las competencias relacionales en cuanto al ámbito y método privilegiado: es la relación que el profesional establece con los futuros adoptantes, con el otro profesional, con los demás miembros del equipo supra-zonal de referencia, con los jueces del Tribunal de los Menores. Se encuentran en este primer grupo las siguientes indicaciones: menor ansiedad de prestación (12), más flexibilidad en la interpretación del esquema proporcionado por el Tribunal de los Menores de edad (5), la relación con la pareja adoptiva es más auténtica y refinada (5), más atención a las consecuencias de su propia labor (5). 
El segundo grupo de citas se refiere a las competencias relativas a los contenidos y a los instrumentos de análisis y, por lo tanto, al mayor o menor grado de capacidad adquiridos por el profesional en el tiempo, según su propio juicio. Forman parte de este grupo: una actitud de mayor rigor que puede transformarse también en mayor rigidez en la valoración de las parejas (16); una mayor profundidad de análisis - conseguida a través del aprendizaje, de la experiencia y de los errores, de la formación y de la adquisición de instrumentos más finos (35); una ampliación del espectro de temas útiles al análisis de la relación adulto-niño (conocimiento más profundizado de fenómeno del abuso infantil, de las relaciones en la familia ampliada, de los sufrimientos típicos de los menores abandonados (6). Sólo dos menciones se refieren a una menor motivación en el trabajo del profesional en las adopciones - debida al aburrimiento (1) y a la declaración que nada cambió (1). Es más, muchos destacan la importancia de la comparación continua entre profesionales. Pues, los profesionales ofrecen una representación de ellos mismos como menos preocupados por la prestación en comparación a un tiempo, como más rigurosos en respectar el mandato de la autoridad judicial (hasta asumir una actitud más rígida en la ejecución de la tarea), como más capaces de valorar, describir, argumentar, analizar de forma más esmerada que en el pasado.

\section{ConClusiones}

La investigación presentada en esta contribución ha pretendido reconstruir la experiencia madurada por los profesionales que actúan en la región italiana de Piamonte -veinte años después de la institución de los equipos de adopción- a través del estudio de los procesos de evaluación de las parejas que quieren adoptar a un menor abandonado. El objeto del análisis está constituido por los temas indagados, por los criterios de evaluación y por las representaciones difusas e implícitas que influencian constantemente los pensamientos y las acciones de los profesionales implicados en esos procesos. Por dicho motivo se ha tenido en gran consideración la reconstrucción del pensamiento de los entrevistados respecto a las razones de su propia conducta y a la estructuración de sus intervenciones. Aunque todavía se perciba un dilema no solucionado entre dos polaridades, es decir entre conocimiento y valoración de la pareja, se debe señalar que en los últimos años los coloquios de consulta-valoración empiezan a perder la connotación

Mediaciones Sociales, № 10, I semestre 2012, pp. 89-117. ISSN electrónico: 1989-0494. DOI: http://dx.doi.org/10.5209/rev_MESO.2012.n10.39683 
de coloquios de selección o encuentros de evaluación, adquiriendo el carácter de encuentros de orientación-maduración. Son encuentros que acompañan a la pareja en la reflexión sobre el sentido de la acogida de un menor que ha sufrido, orientados a potenciar recursos dentro de la familia, hacer madurar motivaciones idóneas para la elección adoptiva o, posiblemente, acompañar a la pareja en la serena decisión de desistir del empeño adoptivo. Tanto las respuestas al estímulo de la breve narración que abrió la entrevista (apartado 5), como los demás datos confirman la conciencia de los expertos acerca de su propia responsabilidad en cuanto al resultado de la valoración sobre la idoneidad de las parejas. También desprende un fuerte sentido de pertenencia a la organización y al orgullo de hacer correctamente su trabajo.

Los resultados evidencian una gran atención a los tres temas sobre los que se basa la disponibilidad a la parentalidad adoptiva: las modalidades de funcionamiento de la relación de pareja, la propensión a la parentalidad, la atención al menor y a las consecuentes necesidades de cuidado. Por lo tanto, el empeño prodigado por los profesionales propende a la búsqueda de todos los elementos útiles a evidenciar comportamientos y actitudes coherentes por parte de cada adoptante en el paso de pareja a padres adoptivos, incluyendo acontecimientos y pasos dolorosos que recordar, o considerados especialmente íntimos.

Por cuanto atañe a los interrogantes cognitivos subtendidos a los objetivos de investigación, los resultados presentan algunas respuestas interesantes en cuanto a la influencia de las representaciones de parentalidad biológica y parentalidad adoptiva $\mathrm{y}$ a las eventuales diferencias presentes entre la adopción nacional y la internacional, con respecto a las decisiones asumidas por los profesionales.

En primer lugar, las respuestas otorgadas a los estímulos del diferencial semántico han demostrado que los contenidos de representación de la parentalidad biológica y de la adoptiva son casi totalmente superpuestas, tanto para los psicólogos como para los asistentes sociales. Dicho resultado demuestra que entre los profesionales existe el mismo horizonte de sentido imputable a una parentalidad compuesta de disponibilidad afectiva, de sentido de responsabilidad y de conciencia del papel de guía al respeto de las reglas, sin presentar particulares diferencias entre adopción nacional e internacional. 
Además, en la segunda sección de la entrevista se ha pretendido detectar cuáles son los temas afrontados y cuáles los criterios de valoración en el proceso de evaluación-orientación de las parejas adoptivas. Ha sido, este, un paso importante en la impostación y en la realización de la investigación, porque ha permitido confrontar cuánto afirmado por los profesionales con sus representaciones del proceso, de los temas y de los criterios utilizados.

Los resultados conseguidos demuestran que la gran parte de los profesionales suelen sobreponer criterios y temas, y también criterios e instrumentos de intervención. Actuando así cada experto se somete a un nivel de estrés decisional mayor que si se refiriera a un sistema de valoración basado en criterios comunes y consolidados. Lo afirmado, aparece aún más evidente en correlación con otro de los dilemas que tradicionalmente caracterizan el trabajo en campo adoptivo, o sea la elección entre la importancia central del menor con respecto a la de la pareja adoptante. En efecto, muchas respuestas a las preguntas de la entrevista y a los focus groups, han destacado la dificultad en cumplir con los dictámenes legislativos que prescriben la importancia del menor. Además, muchos han matizado la importancia de la confrontación continua entre profesionales, a menudo señalado como único instrumento de comparación entre puntos de vista distintos.

Es más, para notar la pertinencia de los criterios estimativos con las transformaciones sociales de las familias, ha sido además indagado el eventual cambio ocurrido en los modos de estudiar a los aspirantes a la adopción. Los psicólogos y los asistentes sociales piamonteses han afirmado que lo que ha ocurrido no ha sido un cambio de criterios de evaluación, sino que un cambio en las modalidades de su aplicación. Pues, los profesionales han ofrecido una representación de ellos mismos como menos preocupados por la prestación; como más rigurosos por respetar el mandato de la autoridad judicial; como más capaces de evaluar, describir, argumentar, analizar de forma más esmerada que en el pasado. También se ha manifestado una fuerte tendencia a afrontar todos los temas contemplados por las disposiciones legislativas acerca del recorrido que los profesionales y las parejas deben actuar. En particular, parece evidente el empeño de asistentes sociales y psicólogos por sondear las motivaciones, la disponibilidad a acoger la diversidad y las modalidades de funcionamiento de la potencial pareja parental adoptiva. Los profesionales averiguando si aquella pareja es capaz de aceptar el desafío

Mediaciones Sociales, № 10, I semestre 2012, pp. 89-117. ISSN electrónico: 1989-0494.

DOI: http://dx.doi.org/10.5209/rev_MESO.2012.n10.39683 
lanzado de la parentalidad adoptiva, logran integrar aspectos de identidad y semejanza virtual con los de diferencia.

En conclusión, lo que se quiere evidenciar en esta sede es la extrema utilidad de proceder individuando criterios de evaluación y descriptores compartidos en base a la experiencia consolidada e respaldados por el método científico.

Digno de nota es el esfuerzo efectuado por algunas Regiones italianas en producir líneas de orientación para la valoración psico-social de la pareja. Son instrumentos funcionales para homogeneizar las intervenciones de los profesionales y, sin embargo, si no están acompañados por criterios validados a nivel empírico y por descriptor útiles al proceso decisional de los profesionales y de los jueces, corren el riesgo de acabar siendo una lista puramente descriptiva. La importancia de este estudio reside también en la tentativa de concurrir al conocimiento del fenómeno adoptivo: es evidente que una esmerada evaluación inicial de las parejas aspirantes a la adopción puede prevenir fracasos futuros de acoplamiento. Sin embargo, no pasa inadvertido un límite ínsito en este estudio, es decir que la estructura de la investigación prevé el análisis de la perspectiva de los profesionales, pero no la de los padres adoptivos y de los hijos adoptados, que podrá incluirse en una próxima investigación.

\section{BibLIOGRAFÍA}

Ainsworth, M. D., Blehar, M. C., Waters, E., Wall, S. (1978): Patterns of Attachment: a Psycological Studi of the Strange Situation. Hillsdale: Erlbaum.

Allegri, E. (1999): "Postfazione", in De Rienzo, E., SAccoccio, C., Tonizzo, F., Viarengo, G.: Storie di figli adottivi. Torino: UTET.

Allegri, E., Favretto, A. R., Vaiuso, D., Bella, G. (2006): Vent'anni di adozione in Piemonte, Rapporto di ricerca, Dipartimento di Ricerca Sociale, Università del Piemonte Orientale “A. Avogadro” e Regione Piemonte, novembre 2006.

Berger, P., Luckmann, T., (1969): La realtà come costruzione sociale. Bologna: Il Mulino.

BowlBy, J. (1989): Una base sicura. Milano: Cortina.

Mediaciones Sociales, № 10, I semestre 2012, pp. 89-117. ISSN electrónico: 1989-0494.

DOI: http://dx.doi.org/10.5209/rev_MESO.2012.n10.39683 
Cigoli, V. (1992): Il corpo familiare. Milano: Angeli.

Fava Vizziello, G. M., Simonelli, A. (2004): Adozione e cambiamento. Torino: Bollati Boringhieri.

Fivaz-Depeursinge, E., Corboz-Warnery, A. (2000): Il triangolo primario. Milano: Cortina.

GoffMan, E. (1969): La vita quotidiana come rappresentazione. Bologna: Il Mulino.

Greco, O., Ranieri, S., Rosnati, S. (2003): Il percorso della famiglia adottiva. Strumenti per l'ascolto e l'accompagnamento. Milano: Unicopli.

GuIDI, D., CANTÙ, D. (2000): “Alla ricerca della genitorialità: perché non basta il desiderio per diventare genitori adottivi”, Minori e Giustizia, n ${ }^{\circ}$, pp.46-53.

Malagoli Togliatti, M., Mazzoni, S. (2006): Osservare, valutare e sostenere la relazione genitoriale. Milano: Cortina.

Mchale, J. P., Kuersten-Hogan, R., Lauretti, A. (2006): "Valutazione della co-genitorialità e delle dinamiche a livello familiare durante la prima e la seconda infanzia: il sistema di codifica della co-genitorialità e della famiglia”, in KERIG, P., LindHAL, K. M. (eds.): Sistemi di codifica per l'osservazione delle relazioni familiari, Milano: Angeli.

Osgood, C. E., Suci, G. J., Tannenebaum, P. H. (1957): The measurement of meaning. Urbana: University of Illinois.

SCABini, E., Cigoli, V. (2000): Il famigliare. Milano: Cortina.

Zerilli, M., Rosa Volpe, B. (2004): "I follow-up", in Fava Vizziello, G., Simonelli, A.: Adozione e cambiamento. Torino: Bollati Boringhieri, pp. 312-333.

Legge 4 maggio 1983, n. 184, “Disciplina dell'adozione e dell'affidamento dei minori”.

Convenzione 29 maggio 1993, "Sulla protezione dei minori e sulla cooperazione in materia di adozione internazionale”, Aja.

Legge 28 marzo 2001, n.149, "Modifiche alla legge n.184, que lleva "Disciplina dell'adozione e dell'affidamento dei minori”, así como en el título "VIII del libro primo del codice civile”. 


\section{PARA CITAR ESTE TRABAJO EN BIBLIOGRAFÍAS:}

ALLEGRI, Elena (2012): "Entre certezas y dilemas: investigación sobre la experiencia de los equipos de adopción en Italia”, Mediaciones Sociales. Revista de Ciencias Sociales y de la Comunicación, $\mathrm{n}^{\circ}$ 10, pp. 89-117. DOI: http://dx.doi.org/10.5209/rev_MESO.2012.n10.39683

\section{${ }^{(*)}$ La autora}

Elena Allegri es investigadora y profesora de Sociología y Trabajo Social en la Universidad de Piamonte Oriental en Alessandria, Italia. Es presidenta del curso de licenciatura trienal en Trabajo Social y forma parte del Colegio de Profesores del Doctorado en Sociología y Metodología de Investigación Social de la Universidad Bicocca de Milan y de la Universidad de Piamonte Oriental, Italia. Es autora de numerosos ensayos sobre trabajo social, mediación familiar y estudio de las representaciones sociales. Ha publicado: Supervisione e lavoro sociale (1997), La mediazione familiare. Temi e ricerche (2004), Il colloquio nel lavoro sociale (2006), Le rappresentazioni dell'assistente sociale. Il lavoro sociale nel cinema e nella narrativa (2006). Ha participado en el Comité Científico del primer Diccionario italiano de Trabajo social. Ha dirigido proyectos de supervisión e investigación-intervención para numerosas organizaciones sociosanitarias.

RECIBIDO: 15 de septiembre de 2011.

ACEPTADO: 30 de marzo de 2012. 
